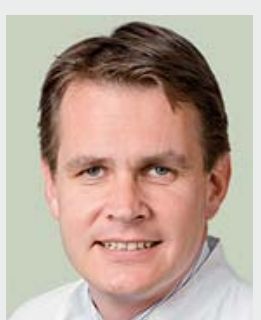

Ulrich Stöckle

\title{
Editorial für das OP-Journal
}

\author{
Liebe Leserin, lieber Leser,
}

das OP-journal hat in den letzten Jahren mit Themenheften immer wieder den aktuellen Versorgungsstand in einzelnen Bereichen unseres Fachgebietes dargestellt. Die hohe Qualität der Beiträge von namhaften Autoren aus unterschiedlichen Kliniken ist dabei äußerst bemerkenswert.

Das aktuelle OP-Journal beschäftigt sich mit knöchernen und Weichteilverletzungen rund ums Kniegelenk.

Insbesondere im Bereich der Tibiakopffrakturen hat sich in den letzten Jahren ein neues Verständnis entwickelt, basierend auf einer CT-gestützten Klassifikation, die eine Unterscheidung von 10 Frakturbereichen ermöglicht. Dies hat Konsequenzen für die Zugangswahl und Stabilisierungsoptionen.

Insbesondere Kombinationsverletzungen, wie Luxationsfrakturen, erschweren die Therapie, die nach klaren Standards erfolgen sollte. Die Versorgung von distalen Femurfrakturen und auch Patellafrakturen sowie Frakturen mit schweren Weichteilschäden vervollständigen das Spektrum, ergänzt um die plastischen Rekonstruktionsmöglichkeiten rund um das Kniegelenk.

Mit der vorliegenden Ausgabe des OP-Journals hoffen wir, den aktuellen Stand der Versorgung von knöchernen und Weichteilverletzungen rund ums Kniegelenk darstellen zu können. Besonderer Dank gilt den namhaften Autoren für ihre qualitativ hochwertigen Beiträge. Falls Sie die in dieser Ausgabe angesprochenen Versorgungsprinzipien von Verletzungen rund ums Kniegelenk weiter vertiefen möchten, verweisen wir gerne auf die von der AOTrauma Deutschland angebotenen Spezialkurse.

Das Präsidium der AOTrauma Deutschland ist der Herausgeber des OP-Journals. Daher möchte ich an dieser Stelle Michael Raschke und Florian Gebhard für die gute Zusammenarbeit in den letzten 6 Jahren ganz herzlich danken.

Durch die Neubesetzung des Präsidiums der AOTrauma Deutschland haben Ulf Culemann und Christof Müller auch die Position der Herausgeber des OP-Journals übernommen.

Mit freundlichen Grüßen

Prof. Dr. med. U. Stöckle

Präsident AOTrauma Deutschland

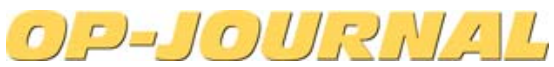

mitbegründet von

Prof. Dr. med. Günther Hierholzer

Prof. Dr. med. h. c. mult. Siegfried Weller

Herausgeber

AOTrauma Deutschland e. V.

Präsident: Prof. Dr. med. Ulrich Stöckle, Berlin
Schriftleiter

Prof. Dr. med. Ulf Culemann, Celle

Prof. Dr. med. Christof Müller, Karlsruhe

Prof. Dr. med. Ulrich Stöckle, Berlin

Beirat

Priv.-Doz. Dr. med. Hermann Josef Bail, Nürnberg

Prof. Dr. med. Karl-Heinz Frosch, Hamburg

Priv.-Doz. Dr. med. dent. Alexander Gröbe,

Hannover
Prof. Dr. med. Marius Johann Baptist Keel, FACS, Bern, Schweiz

Prof. Dr. med. Dr. h. c. Edgar Mayr, Augsburg Prof. Dr. med. Wolfgang Lehmann, Göttingen Prof. Dr. med. Hans-Christoph Pape, Zürich Prim. Dr. med. Michael Plecko, Graz, Österreich Prof. Dr. med. Klaus Schaser, Dresden Alexander Motzny, Tübingen 